\title{
THE CREATION OF FLOOD RISKS MODEL USING A COMBINATION OF SATELLITE AND METEOROLOGICAL MODELS - THE FIRST STEP
}

\author{
Monika BIRYLO $^{1) *}$, Jolanta NASTULA ${ }^{2)}$ and Joanna KUCZYNSKA-SIEHIEN ${ }^{1)}$ \\ 1) University of Warmia and Mazury, Department of Surveying and Geoscience, Heweliusza St. 12, 10-724 Olsztyn \\ ${ }^{2)}$ Space Research Center Polish Academy of Science, Bartycka St. 18A, 00-716 Warsaw \\ *Corresponding author's e-mail: monika.sienkiewicz@uwm.edu.pl
}

\section{ARTICLE INFO}

Article history:

Received 2 February 2015

Accepted 1 May 2015

Available online 22 May 2015

\section{ABSTRACT}

An aim of the research is to analyze whether observations from gravimetric GRACE mission and GOCO hydrosphere models can be useful for evaluating local hydrosphere conditions, and so, flood and drought prediction. The investigation shows that combined gravimetric and meteorological models provide more reliable modelling of water flows than "gravimetric only" and "meteorological only" models.

Keywords:

Flood

Hydrosphere

GRACE

GOCO03S

Gaussian filtering

Model combination

\section{INTRODUCTION - THEORETICAL BACKGROUND}

Satellite-borne gravimetric models have a global range, so regionally the accuracy is often not satisfying when precise perception is needed. But using satellite gravimetric data is a cheap and quick way. On the other hand, in geodesy a meteorological data does not fulfill all requirements of a geodetic task. Moreover, in a flood/drainage situation there is a need of quick, cheap, regional and accurate value of equivalent water thickness (EWT). The idea of combining gravimetric and meteorological model was caused by a wish of preparing a EWT model which is the closest to reality.

In geodesy one of the most important issues is the geoid, which link all surveying methods and is a basis of every geodetic tasks. Over the years, scientists elaborated plenty of approaches to determine the geoid. Here, in the paper authors want to present a geoid computation, which is computed in a similar way to EWT using satellite gravimetric data. The computed EWT values changes are used for estimation of water flow risks. In the paper two gravimetric models are used; first based on the GRACE mission observation, and the other is the GOCO03S model, combination of CHAMP, GRACE, GOCO and SLR data. In the paper a comparison of the two models is presented for better and more conscience usage of gravimetric observations. Gravimetric model is then combined with a meteorological data using Gaussian filtering, which has delivered a combined model for flood prediction. from:

Geoid height change by time can be computed

$$
\begin{aligned}
\delta N(\phi, \lambda, t) & =\delta N(\phi, \lambda, t)^{G R A C E}+\delta N(\phi, \lambda, t)^{C}+ \\
& +\delta N(\phi, \lambda, t)^{O}+\delta N(\phi, \lambda, t)^{L},
\end{aligned}
$$

(Veselinova Renglova, 2007)

In the formula, $\delta N(\varphi, \lambda, t)^{G R A C E}$ means geoid change computed with spherical harmonic coefficients $\bar{C}_{l, m}(t)$ and $\bar{S}_{l, m}(t)$ of degree 1 and order $m$. This is calculated by:

$$
\begin{aligned}
& \delta N(\varphi, \lambda, r)= \\
& \quad=R \sum_{l=2}^{\infty} \sum_{m=0}^{l}\left(\bar{C}_{l, m} \cos m \lambda+\bar{S}_{l, m} \sin m \lambda\right) \bar{P}_{l, m}(\sin \varphi),
\end{aligned}
$$

(Chao and Gross, 1987)

The rest of the elements of the formula are errors as follows: $\delta N(\varphi, \lambda, t)^{C}-$ commission error of the involved spherical harmonics, $\delta N(\varphi, \lambda, t)^{O}$ - omission error, and $\delta N(\varphi, \lambda, t)^{L}$ - additional errors, as filtering errors, consequence of the smoothing. All in all, a relation between mass variations and gravitational potential changes is:

$\left\{\frac{\Delta \bar{C}_{l, m}}{\Delta \bar{S}_{l, m}}\right\}=\frac{\delta_{\dot{s} r}(2 l+1)}{3 \delta_{w}\left(1+k_{l}\right)}\left\{\begin{array}{l}\Delta C_{l, m} \\ \Delta S_{l, m}\end{array}\right\}$,

(Wahr et al., 1998) 
Earth mass changes cause gravity change. A relation between spherical harmonics and mass change is as follows:

$$
\left\{\begin{array}{c}
\Delta \bar{C}_{l, m} \\
\Delta \bar{S}_{l, m}
\end{array}\right\}=\frac{1}{(2 l+1) M_{e}} \times \underset{\substack{\text { Masa } \\
\text { Ziemi }}}{\iint}\left(\frac{r^{\prime}}{a_{e}}\right)^{l} \bar{P}_{l, m} \sin \phi^{\prime}\left\{\begin{array}{c}
\cos m \lambda^{\prime} \\
\sin m \lambda^{\prime}
\end{array}\right\} d m
$$

(Wahr et al., 1998)

\section{DATA}

Gravity coefficients $\Delta \mathrm{C}_{1, \mathrm{~m}}, \Delta \mathrm{S}_{1, \mathrm{~m}}$ were computed on a basis of one-stage differential post processing.

\subsection{DATA USED IN A PAPER ARE:}

- GRACE model filtered with Anisotropic NonSymmetric Filter,

- GOCO03S model (a combination of GRACE, GOCE, CHAMP and SLR data),

- Meteorological data in a form of WGHM model.

\subsubsection{GRACE DATA}

Satellites of the mission called Gravity Recovery and Climate Experiment (GRACE) were launched and placed in orbit in 2002, and provide information about the temporal variation of the gravity field of the Earth (Tapley et al., 2004). Satellites are on a low, 500-km orbit at a distance from each other about 220 kilometers - between 170 and $270 \mathrm{~km}$ (Svehla and Foldvary, 2006; Somodi and Foldvary, 2011). Deviations of the gravitational field of the Earth cause a slight change in the distance between the two GRACE satellites. GPS satellites help to determine the precise position of the two GRACE satellites in orbit. On-board accelerometers are used to measure the non-gravitational acceleration (Tapley et al., 2004).

CSR Data Centers have latest been delivered the RL-05 (Release Note 05) models based on the GRACE measurements. Solutions of the various centers are different because of the degree of the spherical harmonics expansion - ranging from a degree of 60 or more. GRACE gravity field models are published as GSM files (Bettadpur, 2007). They contain $\Delta \mathrm{C}_{\mathrm{l}, \mathrm{m}}(\mathrm{t})$ and $\Delta \mathrm{S}_{\mathrm{l}, \mathrm{m}}(\mathrm{t})$ coefficients of the gravity field expansion into spherical harmonics series (Bettadpur, 2007). From the previous products the RL-05 differs notably, since the used tidal and static field models are more accurate (Watkins et al., 2007).

In case of the available GRACE models, a filtering over area of some $100 \mathrm{~km}$ is unavoidable due to overcome the stripes in the raw data (Foldvary, 2007). The easiest way to remove errors of a higher degree of spherical harmonics is a simple filter: apply limited maximal degree of spherical harmonics expansion (Sneeuw et al., 2005). This approach has the disadvantage that it greatly reduces the signal strength, despite the removal of the systematic errors (Templey and Reiger, 2008).
GRACE RL05 data were acquired from the CSR GRACE website in a form of extension of the spherical harmonic coefficients $\Delta \mathrm{C}_{1, \mathrm{~m}}, \Delta \mathrm{S}_{\mathrm{l}, \mathrm{m}}$. To compute a millimeters of EWT with filtration from raw GRACE observation, Anisotropic NonSymmetric Filter (ANS) was used, described in equation below.

$$
f_{x}(x)=\frac{1}{4 \Pi R^{2}} \sum_{n=0}^{N}(f(y) W(x, y))
$$

Where:

(Zhang, 2009)

$\mathrm{f}_{\mathrm{w}}$ - mass change, $\mathrm{R}-$ Earth radius and a filter $\mathrm{W}(\mathrm{w}, \mathrm{y})$ describes formula:

$$
W(x, y)=\sum_{l, m} \sum_{p, q} W_{i m, p q} Y_{l, m}^{\prime}(\xi) Y_{p, q}^{\prime}(\eta)
$$

Where $1, \mathrm{~m}$ are degree and order of a spherical harmonic expansion, Y'l,m normalized spherical harmonic, Wlm,pq filtering coefficient, and $\zeta$ and $\eta$ coordinates of a point on a sphere.

ANS filter in non-symmetric in accordance to points $x, y$ and is dependent on both, degree and order. Moreover, it can minimize root mean square error (Klees et al., 2008).

\subsubsection{GOCOO3S MODEL}

The second model was GOCO (Gravity Observation Combination), in its version of $03 \mathrm{~S}$ (Mayer-Gurr et al, 2012). The aim of GOCO model preparation was to achieve a global gravity field model of a great accuracy and resolution. It is a combination of CHAMP, GRACE, GOCE and SLR previously individually for each filtered observations. A terrestrial data is also added. Methodology of evaluating a GOCO model was superposition of full normal equations plus optimal weighting of the mentioned models. In a process of preparing a GOCO03S model, at first it is parameterized, than a combination of a consistent normal equation is computed, at least an optimum solution is prepared in a form of variance component estimation. Up to 120 degree and orders GRACE observations are dominant, at 140-220 the GOCE data is dominant, up to 230 the most important role is played by the regularization. This process is connected with errors in extension of the spherical harmonics coefficient. (Goiginger et al., 2011)

\subsubsection{WGHM MODEL}

WaterGAP Global Hydrological Model (WGHM) is a meteorological model that contains data in the form of a half-degree grid. WGHM data are published every month, made up the variables on precipitation, number of rainy days in the month, temperature and cloud cover. The most important parameter is precipitation, because this just has a significant impact on the water cycle. Currently, data are available for the period: 1951.1 to 2005.12 


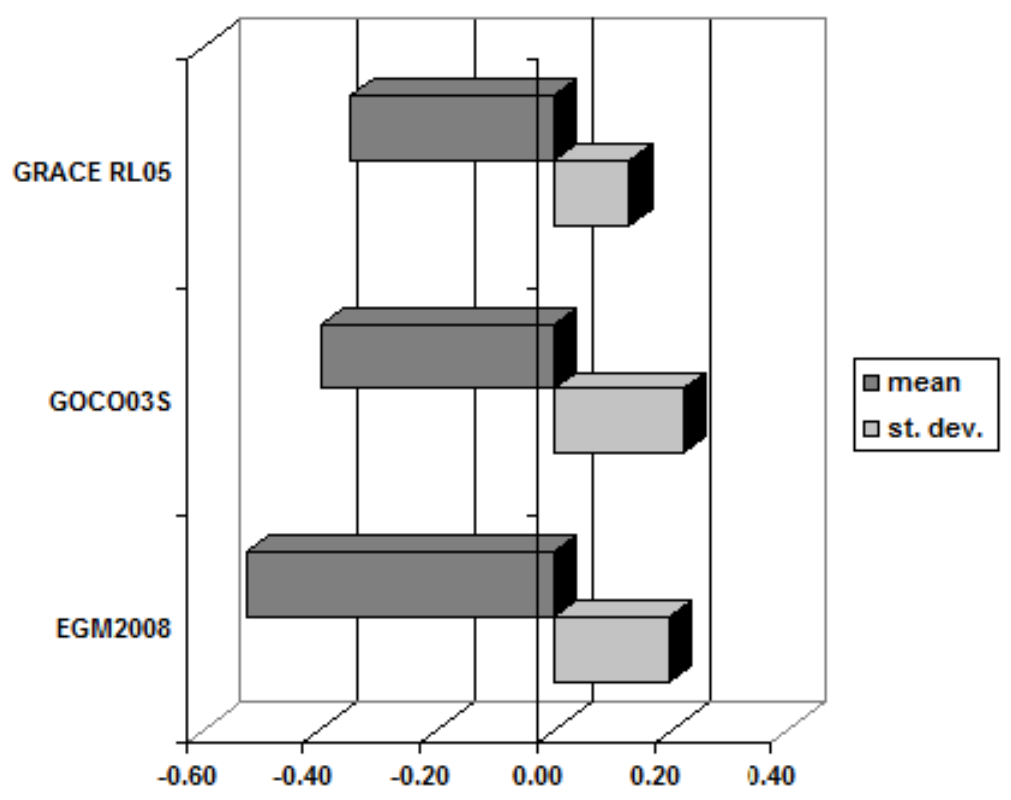

Fig. 1 Statistics of the used gravimetric models.

(Fiedler and Doll, 2007). WGHM is a model defining the richness and use of water resources, there is a combination of water changes due to natural processes and human activities. The model consists of four components: surface water, snow, groundwater, soil, all of which were used in tests (Gunter et al., 2007).

\subsection{AREA: CASE STUDY FOR THE SOUTHERN PART OF POLAND (SUDETY, MALOPOLSKA AND SILESIA AREAS) FOR YEARS 2003-2010}

In a paper two gravimetric models were tested: GRACE observations model (observation from CSR Center webside, filtered by authors with ANS filter) and GOCO03S. The two models, after filtering, were compared to well-known EGM2008 model (Pavlis et al., 2012). The authors decided to compute a gravity anomaly, geoid and deflection of the vertical for GRACE, GOCO03S and EGM2008 models using $\Delta \mathrm{C}_{\mathrm{l}, \mathrm{m}}$ and $\Delta \mathrm{S}_{1, \mathrm{~m}}$. For a better perception of the models, statistical characteristics were put under comparison; mean value and standard deviation were computed. Results are presented in Figure 1. Comparison should have some kind of release model, which can be the same for GRACE, GOCO and EGM2008 model. The authors decided to use POLREF data, not in use in Poland now but still good for tests and accuracy evaluation. POLREF data consists of 348 geodetic points placed in a dispersed but regular network in the whole area of Poland (Pażus, 2009).

It is shown in figure 1 that the least standard deviation when compared to POLREF network was found for the newest GRACE release: RL05 (st. dev.: $0.13 \mathrm{~mm}$ ), standard deviation of GOCO03S and
EGM2008 were almost the same $(0.22 \mathrm{~mm}$ for GOCO03S and 0.20 for EGM2008 mm). It can be said that using GRACE data improved almost two times the accuracy of gravimetric data in Poland. The GOCO03S model's accuracy is almost the same as that of EGM2008. For GRACE RL05 the computed values of EWT were 0.12 , which means that millimeters of EWT computed from the CSR GRACE model were closer to each other in comparison with GOCO03S and EGM2008 models.

\section{MODEL COMBINATION}

The aim of the paper was to try to improve global gravimetric data in such a way that regional applications may take a benefit of it. For this purpose, there was a trial of combining gravimetric and meteorological data. As the meteorological model needs some calibration so that it has the same reference as gravimetric data, some post-processing was expected to be done. The model was previously used; results were described in a paper (Birylo and Nastula, 2013). In that paper the modification and calibration of WGHM was presented, which model correlates the best with gravimetric data. A need of modification detected: a new scaling factor and a twomonth shift has been observed in the meteorological data when compared with gravimetric data in a form of millimeters of EWT.

WGHM model was modified with EWT modification factor (a value of 0.0065 was determined by Birylo, Nastula (2013)). Modification factor was computed according to the formula:

$$
\sum_{i}^{N}\left(S_{i}^{P}-A S_{i}^{F}\right)^{2}
$$




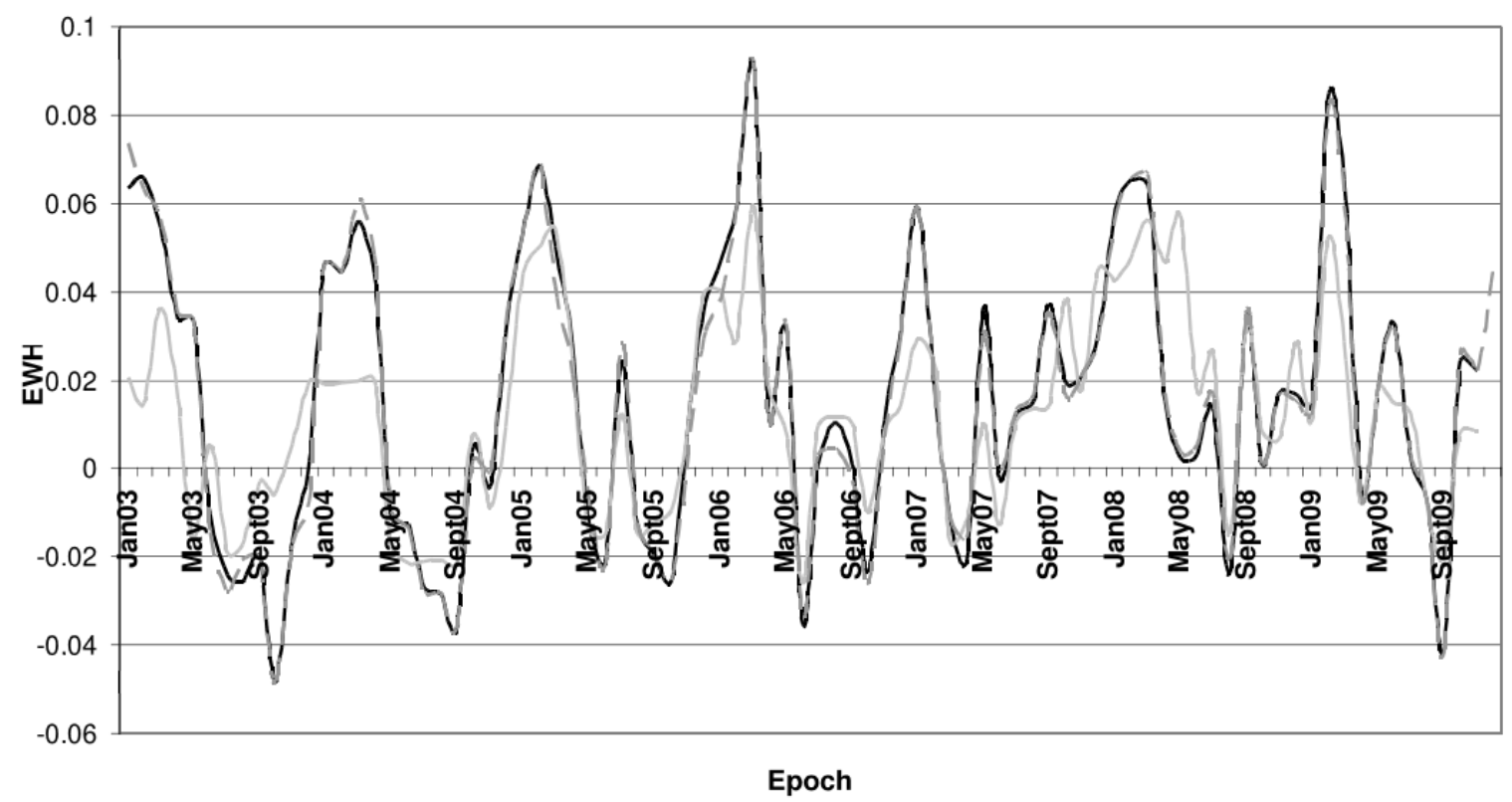

Fig. 2 Comparison between GRACE, GOCO03S and WGHM data.

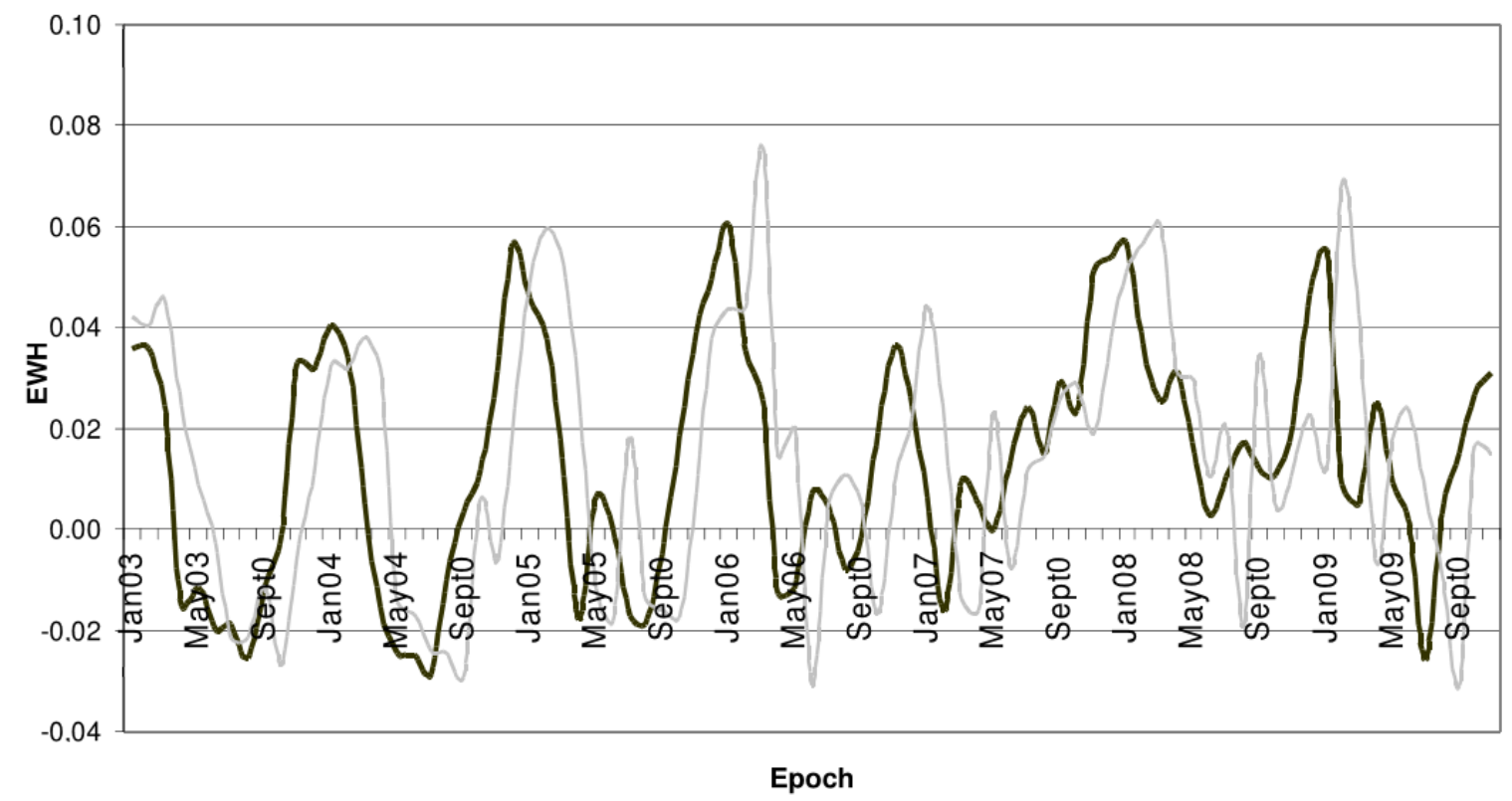

Fig. 3 Combination GRACE+WGHM compared to the original CSR GRACE RL05 model (darker) and GOCO+WGHM compared to the original GOCO03S model (lighter).

Where $S_{i}^{P}$ and $S_{i}^{F}$ are EWT values of each model $(P$ for the first reference model, which was taken into account, while $F$ index refers to the following model), $N$ is the number of months, and $A$ is the scale factor.

Results are presented in Figure 2, where dark line describes ETW for southern Poland from GRACE data; dashed light line describes ETW for southern Poland from GOCO03S model and light line is the WGHM model.
The next step was combining gravimetric data with meteorological data. For this purpose a spherical harmonic coefficients were set in a way: GRACE + WGHM model and GOCO03S + WGHM model, finally a combined modell using Gaussian filtering (Jekeli, 1981). Results are presented in Figure 3: the difference between original CSR GRACE RL05 model and combined GRACE + WGHM model ( the darker line in Figure 3) and difference between original GOCO03S model and combined GOCO03S + WGHM (lighter line in Figure 3). 
Combination was delivered by using Gaussian filtering. For the data combined in the article some statistics were prepared and evaluated. The computed standard deviation for combined models is as follows:

- For GRACE RL05+WGHM in comparison with GRACE RL05 data: $0.23 \mathrm{~mm}$

- For GOCO03S+WGHM in comparison with GOCO03S: $0.24 \mathrm{~mm}$

Here it should be mentioned that accuracies of the models are: for GOCO03S model $0.407 \mathrm{~mm}$, for CSR GRACE RL05 model $0.220 \mathrm{~mm}$. For comparison, the same value for the EGM2008 model is $0.217 \mathrm{~mm}$ [after ICGEM]. Accuracy of the WGHM model is few millimeters (Flury et al., 2006).

\section{CONCLUSIONS:}

In the paper the authors presented an analysis for combining gravimetric with meteorological models, which is a first step for combining plenty of models in order to derive a water flow risk map. For this purpose few tests were provided:

- a comparison of GRACE, GOCO and EGM2008 models to the POLREF network for a better perception of the accuracy of the used models (gravity anomaly, geoid and deflection of the vertical were computed using spherical harmonic coefficients of GRACE, GOCO and EGM2008, and the statistical characteristics were provided),

- a comparison between GRACE, GOCO and WGHM models in a form of millimeters of EWT in time series,

- two models with Gauss filter applied were prepared: WGHM+GRACE and WGHM+GOCO.

On a basis of the research presented in this paper several conclusions can be drawn. When comparing three models: GRACE, GOCO and EGM2008 with POLREF network, the following statistics were gained: for the GRACE RL05 model it is 0.13 , for GOCO 0.20, and for EGM2008 it was found to be 0.22 . It can be said that GRACE model shows the best agreement in validation to the POLREF network. Moreover, we notice that used WGHM meteorological model needs an additional calibration (modification factor: 0.0065). After combining two models, statistical characteristics were computed; standard deviation of the combined models GRACE+WGHM and GOCO+WGHM both were around 0.20 .

All in all, it can be said that the in first step of model combination, accuracy and statistical characteristics of WGHM model combined with gravimetric model suggest that it is possible to predict flood and drainage periods. Moreover, the method of combining meteorological and gravimetric models, described in the article is universal, can be used in any area, still there is a need of addition of geological data.

\section{ACKNOWLEDGEMENTS}

The work was supported by the National Science Centre, contract number UMO-2012/07/N/ST10/03275 of July 11, 2013.

\section{REFERENCES}

Beerer, J.G. and Massmann, F.H.: 2007, Status GRACE Mission Operations. GRACE Science Team Meeting, Potsdam, conference proceedings.

Bettadpur, S.: 2007, Level-2 Gravity Field Product, User Handbook, PO. DAAC GRACE Documentation.

Birylo, M. and Nastula, J.: 2013, Regional equivalent water thickness determination as a source of data for flood phenomenon observation. Papers on global change, 19(1), 43-53.

Flury, J., Rummel, R., Reigber, Ch., Rothacher, M., Boedecker, G. and Schreiber, U.: 2006, Observation of the Earth system from space, Springer, 120-128.

Foldvary, L.: 2007, Accuracy issues about the GRACE determined seasonal variations of the gravity field. Geodezia es Kartografia, 59(8-9), 40-46.

Chao, B. F. and Gross, R. S.: 1987, Changes in the Earth's rotation and low-degree gravitational field induced by earthquakes. Geophys. J. R. astr. Soc. 91, No. 3, 569596. DOI: $10.1111 / j .1365-246 X .1987 . t b 01659 . x$

Goiginger, H., Hoeck, E., Rieser, D., Mayer-Gurr, T., Majer, A., Krauss, S., Pail, R., Fecher, T., Gryuber, T., Brockmann, J.M., Krasbutter, I., Schuh, W.D., Jaeggi, A., Prange, L., Hausleitner, W., Baur, O. and Kusche, J.: 2011, The combined satellite only global gravity model GOCO02S, EGU, Vienna, Austria April, 4-8, 2011, conference proceedings.

Gunter, A., Stuck, J., Werth, S., Doll, P., Verzano, K. and Merz, B.: 2007, A global analysis of temporal and spatial variations in continental water storage. Resources Research (AGU), 43, W05416.

DOI: 10.1029/2006WR005247

Jekeli, C.: 1981, Alternative methods to smooth the Earth's gravity field. Report No. 327, Geodetic and GeoInformation Science, Department of Civil and Environmental Engineering and Geodetic Science, Ohio State University.

Klees, R., Liu, X., Wittwer, T., Gunter, B.C., Revtova, E.A., Tenzer, R., Ditmar, P., Winsemius, H.C. and Savenije, H.H.G.: 2008, A comparison of global and regional GRACE models for land hydrology: Survey Geophysics., 29, 335-359. DOI: $10.1007 / \mathrm{s} 10712-008-9049-8$

Mayer-Gürr, T. et al.: 2012, The new combined satellite only model GOCO03S, Abstract submitted to GGHS2012, Venice (Poster), conference proceedings.

Pavlis, N. K., Holmes, S. A., Kenyon, S. C. and Factor, J. K.: 2012, The development and evaluation of the Earth Gravitational Model 2008 (EGM2008). Journal of Geophysical Research: Solid Earth (1978-2012), 117, B4. DOI: 10.1029/2011JB008916

Pażus, R.: 2009, Multi-functional system of precise satellite positioning ASG-EUPOS, National Reference System, part 1: reference frames and systems in Poland (in Polish). Published by the National Office of Geodesy and Cartography, Department of Geodesy, Cartography and Geographic Information Systems, avalilable online: asgeupus.pl.

Sneeuw, N, Gerlach, Ch, Foldvary, L, Gruber, Th, Peters, Th, Rummel, R. and Svehla, D.: 2005, One year of 
time-variable CHAMP-only gravity field models using kinematic orbits. In: A Window on the Future of Geodesy, IAG Symposia, 128. (Ed. Sanso, F), Springer-Verlag, Berlin Heidelberg, 288-293.

Somodi, B. and Foldvary, L., 2011: Application of numerical integration techniques for orbit determination of state-of-the-art LEO satellites. Periodica Polytechnika Civil Engineering, 55/2, 99106.

Svehla, D. and Foldvary, L.: 2006, From kinematic orbit determination to derivation of satellite velocity and gravity field. In: Observation of the Earth System from Space ( Flury, J., Rummel, R., Reigber, C., Rothacher, M., Boedecker, G. and Schreiber, U. (eds.)), Springer Berlin Heidelberg New York, 177192.

Swenson, S. C. and Wahr, J.: 2006, Post-processing removal of correlated errors in GRACE data. Geophysical Research Letter, 33, Issue 8, L08402. DOI: $10.1029 / 2005$ GL025285.

Tapley, B., Bettadpur, S., Ries J., Thompson, P. and Watkins, M.: 2004, GRACE measurements of mass variability in the Earth system. Science Magazine AAAS, 23, 5, 503-505.

DOI: $10.1126 /$ science. 1099192

Veselinova Renglova, E.: 2007, A Dynamic Geoid Model for Canada. A thesis submitted to the Faculty of graduate studies in partial fulfillment of the requirements for the degree of doctor of philosophy. UCGE Reports Number 20261, 18 pp.

Wahr, J., Molenaar, M. and Bryant, F.: 1998, Time variability of the Earth's gravity field: hydrological and oceanic effects and their possible detection using GRACE. Journal of Geophysical Research, 103, B12, 30205-30229. DOI: 10.1029/98jb02844

Watkins, M., Gruber, T. and Bettadpur, S.: 2000, Science Data System Development Plan GRACE. Revision C, Information System and Data Center (http://isdc.gfz-potsdam.de).

Zhang, Z.Z., Chao, B.F., Lu, Y. and Hsu, H.T.: 2009, An effective filtering for GRACE time-variable gravity: Fan filter. Geophysical Research Letters, 36, No. 17. 17311. DOI: 10.1029/2009GL039459

http://www.csr.utexas.edu/grace/

http://icgem.gfz-potsdam.de/ICGEM 